\title{
Entre attachements ordinaires et constructions médiatiques dans les quartiers du centre d'Athènes durant la crise
}

Irène Micha et Dina Vaiou

\section{(2) OpenEdition \\ Journals}

Édition électronique

URL : http://journals.openedition.org/pa/435

DOI : $10.4000 /$ pa. 435

ISSN : 2273-0362

Éditeur

Université Lumière Lyon 2

Édition imprimée

Pagination : 166-185

ISBN : 1634-7706

ISSN : 1634-7706

\section{Référence électronique}

Irène Micha et Dina Vaiou, « Entre attachements ordinaires et constructions médiatiques dans les quartiers du centre d'Athènes durant la crise ", Parcours anthropologiques [En ligne], 10 | 2015, mis en ligne le 13 octobre 2015, consulté le 19 avril 2019. URL : http://journals.openedition.org/pa/435; DOI : 10.4000/pa.435 


\section{Entre attachements ordinaires et constructions médiatiques dans les quartiers du centre $d^{\prime}$ Athènes durant la crise}

Irène Micha

Université Technique Nationale d'Athènes, Grèce

Dina Vaiou

Université Technique Nationale d'Athènes, Grèce

\section{INTRODUCTION}

La crise grecque, dite économique, sévissant depuis une période déjà assez longue, a provoqué des changements radicaux qui s'accompagnent de bouleversements sociaux et de transformations urbaines brutales. Depuis 2008, les prestations sociales sont systématiquement élaguées, les salaires et les pensions de retraite s'effondrent, tandis que la tempête de taxes qui s'abat sur les gens rend leur emploi et leur logement de plus en plus précaires. Ces mutations sont beaucoup plus marquantes dans la ville d'Athènes, là où demeure ou transite quotidiennement la moitié de la population du pays, là où un dense et complexe tissu de production a été désagrégé, là où se concentre le pouvoir politique et où l'espace public devient champ de conflits, de revendications, d'exclusions, mais aussi de développement de multiples attachements. À Athènes, les marqueurs de la crise sont désormais visibles, à l'instar des magasins fermés ${ }^{1}$, des bâtiments abandonnés et des infrastructures négligées, des SDF dans les rues; mais certains sont moins visibles comme le chômage, la criminalité, la colère, la dépression et les suicides - situations et incidents presque inexistants au cours de la décennie précédente. De surcroît, l'espérance de vie, qui était l'une des plus élevées de la zone euro, même dans les régions urbaines, a régulièrement baissé ces dernières années, dans la mesure où de plus en plus d'habitants sont déchus de leur droit à la prévention, aux vaccins et aux médicaments ${ }^{2}$.

$131,2 \%$ du total (1986 sur 6377) en mars 2014. INEMY, Rapport annuel du commerce grec, 2014, (en grec), évaluation annuelle des vingt années de la INEMY-E $\Sigma E E$, consultable sur http://www.inemy.gr

2 Des études épidémiologiques du Centre de Recherches universitaires de l'Institut de Santé

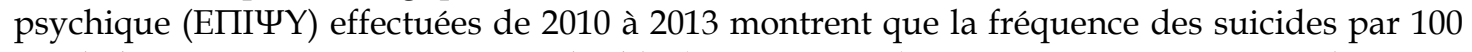
000 habitants en Grèce a presque doublé $(6,5$ contre 3,4$)$. En même temps, on a relevé une augmentation de $20 \%$ des cas de dépression, liée au niveau élevé de difficultés financières 
La crise semble avoir frappé la plupart des quartiers d'Athènes, écrasant quotidiennement la classe moyenne et n'épargnant que ceux qui peuvent encore se soutenir grâce à la richesse accumulée. Pourtant la crise ne frappe pas unilatéralement suivant la stratigraphie de classe sociale. Les intérêts économiques constituent le facteur fondamental de la division sociale, mais il n'est pas le seul (Hall, Massey et Rustin, 2013 : 16). Des exclusions plus ou moins visibles et des inégalités sont suscitées dans l'espace de la ville suivant le sexe, l'origine ethnique, la nationalité, l'âge, la situation de famille, la confession religieuse, la sexualité, et leurs croisements encore plus complexes. Et c'est exactement à ces croisements que l'on distingue aujourd'hui les signes les plus douloureux de la précarité sociale et de la misère. Particulièrement à Athènes, des études sur les retombées socio-spatiales de la crise signalent que l'inégalité la plus marquée est vécue par les immigré(e)s qui habitent les quartiers peuplés et socialement mêlés du centre de la ville (Kandylis, Maloutas et Sayas, 2012). Ainsi, immigré(e)s, femmes au chômage composant des familles monoparentales, personnes âgées, handicapées vivent encore plus profondément l'expérience $\mathrm{du}$ changement brutal modifiant leurs attachements urbains, ce qui les conduit à la marginalité, fait d'eux des citoyens que l'on peut sacrifier tout en les stigmatisant et en leur faisant porter la culpabilité de l'effondrement du pays. Cette lecture, qui souligne les aspects multiples de l'impact économique, démontre que la crise est aussi sociopolitique et surtout idéologique. Elle concerne ainsi les significations et les valeurs socio-spatiales et par conséquent le fonctionnement démocratique de l'État.

Dans ces conditions urbaines instables, l'étude des divers attachements des individus par rapport au cadre spatio-temporel de leur expérience acquiert un intérêt particulier, non seulement afin de cartographier les relations en tant que produits finis d'un processus, mais davantage pour mettre en relief l'ensemble de facteurs qui s'impliquent et se croisent dans ce processus, facteurs qui mêlent la pluralité des temporalités individuelles ou collectives avec leurs différents espaces et les attachements qui singularisent ce rapport au changement et au temps (Botea, 2014: 3). Sous ce prisme, les attachements ordinaires et la réception de l'impact « médiatique » nous aident à mieux comprendre ce qui est aujourd'hui en jeu dans les quartiers du centre $\mathrm{d}$ 'Athènes, à éclairer les différents aspects de la crise, le caractère contingent de ces attachements (Hennion, 2013), la fluctuation, les termes contradictoires et conflictuels de la vie quotidienne et, surtout, les mécanismes qui façonnent et consolident des exclusions. Ces derniers sont des mécanismes depuis toujours incorporés par la ville, mais aussi des mécanismes que les pratiques quotidiennes, historiquement, remettent en question.

(www.epipsy.gr). Voir aussi ELSTAT (Hellenic Statistical Authority), Press Release, 14/05/2015 (www.elstat.gr). 
Dans cet article, nous suivons parallèlement et de manière complémentaire, d'une part, le récit officiel dominant sur le centre d'Athènes tel qu'il s'élabore depuis cinq ans dans le discours politique et celui de la presse ${ }^{3}$; d'autre part, les manifestations variées de pratiques quotidiennes telles qu'elles se développent et s'expriment dans différents quartiers et emplacements sociospatiaux. Tant par les représentations médiatiques de la ville d'Athènes que par les pratiques ordinaires de la vie quotidienne, différents positionnements et attachements se développent et $s^{\prime}$ entremêlent, consacrant des positions sociales ou des rapports de force, traçant des systèmes de valeur et des conventions de comportement, mais formant aussi des conditions de symbiose, de solidarité, d'expression collective et de revendication. Une remarque importante qui découle de l'étude des attachements urbains dans les quartiers du centre d'Athènes est que les récits et les pratiques dominants pénalisent et spolient l'État social et ses infrastructures. En outre, ils détruisent les liens de solidarité qui tentent de fermer les plaies de cet effondrement, du fait que ces liens ne répondent pas à un soi-disant «nous » pur et clair, un "nous » fabriqué et imposé par les médias et le discours politique dominant, procurant des illusions de sécurité.

\section{CONSTRUCTIONS MÉDIATIQUES : RÉCITS DOMINANTS ET SÉCURITÉS FACTICES}

Aujourd'hui, le précepte idéologique dominant de soumission à l'inévitable - absolument indissociable de l'affirmation qu'il n'existe pas d'alternative ${ }^{4}$ limite les droits, fabrique des bipolarisations sociales et retranche des quartiers de la ville, sous le prétexte du retour à une normalité établie (entendue comme idéale et évidente), androcentrée, xénophobe et socialement hiérarchisée. Un discours par conséquent moralisant, dont l'objet est le rétablissement de l'ordre naturel des choses (bouleversé par la crise) par la construction de distinctions socio-spatiales claires et lisibles, sert à un modèle d'autoritarisme gouvernemental inédit. Reposant sur un champ politique troublé depuis les années 1990 et favorisé par les médias, ce discours produit et recycle la peur, consolide la phobie du mélange et ouvre la voie à l'idéologie et à la violence nationaliste et raciste, comme celle de l'organisation néo-nazie Aube Dorée. Chantal Mouffe (2008: 82-84) a souligné que le développement du populisme de droite dans certains pays d'Europe est dû à la fusion de la social-démocratie et de la droite au bénéfice d'une politique de consensus du centre. Par exemple, la prompte montée du Parti de la liberté d'Autriche (FPO) à la fin des années 1990 n'est pas tant due à la nostalgie de l'époque nazie,

3 Notre recherche porte sur la période 2009-2014, c'est-à-dire avant la victoire de Syriza aux élections du 25 janvier 2015.

4 TINA: « There is no alterative ». 
dont résonnaient les formulations du parti, qu'à l'habileté de Georg Heider exploitant l'absence de pluralisme politique, de construire une ligne divisant un pôle vigoureux d'identité collective - le "peuple » - de l'autre pôle qu'il combat, celui des «élites du consensus ». C'est sur des arguments semblables que s'est appuyée aussi la montée du Front National en France des années 1980 jusqu'à aujourd'hui. À une époque où les limites politiques deviennent de moins en moins aisément discernables, Jean-Marie Le Pen soutient qu'il était le seul à remettre en question le consensus dominant. Cette fusion, peutêtre plus que la crise économique, a affaibli en Grèce aussi le fonctionnement démocratique de la vie politique et elle a frayé la voie au remplacement des identités politiques par des identifications de nature religieuse et nationaliste.

Les arguments moralisateurs qui structurent ce discours dominant et les pratiques politiques qui en sont issues, se développent en Grèce progressivement au cours des dernières décennies, et seule leur amplification semble aller de pair avec l'intensification de la crise. En suivant le modèle néolibéral, qui exige d'un côté un environnement urbain plus sévèrement contrôlé, propre et ordonné, capable d'attirer investisseurs et visiteurs, et de l'autre côté un État social moins gaspilleur, la rhétorique politique de la "tolérance zéro », accompagnée d'un programme pour la sécurité en ville reposant sur la répression policière, est apparue en Grèce à la fin des années 1990. A cette époque, les villes grecques ont le taux de criminalité le moins élevé d'Europe (Micha, $2007: 81$ ). La remarque d'Hugues Lagrange (cité dans Bauman, 2008: 38-39) est très significative du fait que les "paniques sécuritaires » les plus spectaculaires ainsi que les plus fortes alertes sur la montée de la criminalité ont eu lieu, dès les années 1960, dans des pays où les subsides de l'État ont été le plus dramatiquement limités (comme aux ÉtatsUnis et en Grande-Bretagne), mais aussi dans des pays où les services sociaux étaient moins développés (comme en Espagne, au Portugal et en Grèce) ${ }^{5}$. Au cours de la période suivante, on constate, comme dans les autres pays du monde occidental, une tendance fortement punitive tant dans les attitudes sociales que dans le cadre institutionnel se rapportant au crime et à son affrontement (Zarafonitou, 2011 : 125). Parallèlement, l'extension du contrôle social s'exprime par l'augmentation du volume du personnel de police. Les patrouilles à pied sont instituées et on entreprend une première cartographie de la « criminalité », qui place dans le viseur les quartiers dangereux de la ville

5 En 2007, à savoir huit années après les premiers chocs de «panique sécuritaire » à Athènes, la Grèce continue à avoir un taux de criminalité inférieur à la moyenne européenne qui la situe à la quatorzième place. Parmi les pays rangés en-dessous de la dix-neuvième place sont : Autriche, France, Portugal, Hongrie et enfin Espagne, qui présente le taux le plus faible de crimes. Ainsi que le remarque Christina Zarafonitou (2009 : 277-8), le paradoxe réside dans le fait que, déjà avant la crise, le sentiment d'insécurité de la société athénienne et sa crainte du crime étaient disproportionnés par rapport au nombre d'incidents enregistrés dans les quartiers de la ville. 
- un titre significatif d'un journal de l'époque est « Athènes : Chicago pour ses habitants » (I Kathimérini, 4/4/1999).

L'extension du droit pénal et le passage simultané à un quotidien d'État policier ont considérablement progressé ces dernières décennies, rencontrant un accueil favorable et un consensus assez large. À cet égard, les Jeux Olympiques de 2004 et le devoir (national) impérieux qu'ils se déroulent dans la sécurité après l'attentat terroriste du 11 septembre 2001 aux États-Unis furent déterminants. Un rôle important fut néanmoins joué par des paramètres endogènes tels que la politique de sécurité tracée parallèlement à cette époque par l'autorité municipale d'Athènes et le pouvoir central et, plus important peut-être encore, par l'absence d'arguments critiques de tous les partis politiques. À cette époque, les brochures électorales des élections municipales et nationales font allusion à des programmes de réhabilitation et de gentrification du centre d'Athènes, ainsi qu'à des équipes d'habitants volontaires organisés pour lutter contre la criminalité 6 . Parallèlement, la « capitale modernisée, multiculturelle et internationalement compétitive » des Jeux Olympiques de 2004 prend grand soin de cacher ses aspects «moins convenables » derrière des panneaux multicolores afin de ne pas exposer aux yeux des visiteurs (et plus encore des téléspectateurs) les marbreries installées depuis plus d'un siècle aux alentours du Stade Olympique, les façades grisâtres des immeubles, les graffiti sur les murs et, dans les quartiers touristiques de la ville, les ajouts architecturaux effectués sans permis de construire - toujours à la faveur de la tolérance de l'État.

Dans le discours officiel ${ }^{7}$ sur les quartiers du centre $\mathrm{d}^{\prime}$ Athènes, s'entremêlent analyses scientifiques et représentations symboliques, mémoires idéologiquement construites, idéalisations, et surtout récits moralisateurs qui font la part belle aux paniques sécuritaires. Après la clôture en fanfare des Jeux Olympiques, on entendit dire que les habitants de la ville avaient abandonné les quartiers du centre pour déménager dans les banlieues plus «vertes et propres». En effet, un pourcentage important de familles aisées recherche depuis les années 1980 de meilleures conditions de vie, principalement

6 En 2004, dans le programme électoral de la formation politique municipale "Athènes demain », alors élue, au chapitre "Action directe pour Athènes ", les trois axes politiques de lutte contre le crime concernent : «...le contrôle plus sévère des délits mineurs commis par les jeunes ayant des tendances à enfreindre la loi [...], la réhabilitation des espaces de la ville qui présentent une image de dégradation et d'abandon et qui peuvent se transformer en foyers de criminalité, et la présence active des habitants de chaque quartier tant par l'intermédiaire de leurs représentants institutionnels que celui de groupes de volontaires » (Athènes Demain, Programme Electoral, 2004).

7 Les informations concernant le discours dominant à Athènes proviennent d'une enquête personnelle effectuée dans la presse grecque quotidienne et hebdomadaire de 2009 à 2014 à l'aide de mots-clés tels que "quartiers du centre ", " sécurité », "criminalité », "ghettos », " régions dangereuses », « immigrés clandestins », « commerce ambulant », etc. Les articles les plus signifiants sont cités dans le texte. 
dans les régions nord-est et littorales situées à l'est et au sud de la capitale. Néanmoins, après 1989, une vague d'émigration (particulièrement d'Albanie et des pays de l'Europe de l'Est) arrive à Athènes, habite dans les appartements vides du centre, travaille dans les chantiers de construction au cours de la période de préparation des Jeux Olympiques et couvre les grands besoins présents dans le champ de l'aide familiale et ménagère. Ces « autres » habitants, auxquels vient s'ajouter une vague d'émigration plus récente (principalement d'Asie et d'Afrique), maintiennent la vie sur les marchés d'Athènes et dans les quartiers du centre même pendant la crise (Vaiou, 2012). On comprend aussi parmi les « autres » invisibles les sans-abris, les drogués, les prostituées et les vendeurs ambulants. Dans les récits dominants, ces groupes non seulement sont discrédités comme habitants, mais ils sont aussi devenus des cibles en tant que responsables de la dégradation sociale (et esthétique). Même si ces groupes sont davantage exposés à la violence et à l'exploitation, ils sont pourchassés par la loi. Les plus récentes cartographies de la criminalité mentionnent désormais les concentrations de sans-abris, les rues de la prostitution, les espaces publics où se fait l'usage de la drogue et d'autres où se développe le commerce parallèle des vendeurs ambulants (identifiés avec les «immigrés clandestins »). Les cartographies sont reliées dans le discours politique à des qualifications et des formulations telles que «zones-ghettos des étrangers » (par le Ministère de la Santé en 2012), « lieux inaccessibles de la ville » (par le maire d'Athènes en 2013), " aucune tolérance envers les immigrés clandestins et le commerce parallèle » (par un député candidat à la mairie d'Athènes en 2014) et des « confirmations » dans des publications analogues : "Le Far West de la rue Aghiou Mélétiou »(Ta Néa 02/11/2012), "Gémissements d'un quartier naguère bourgeois: la délinquance a pris la région au bout de l'avenue Patission » (Ta Néa 12/9/2013).

Ces dernières années, une série de propositions $\mathrm{d}$ 'intervention urbaine vise à la «renaissance » des quartiers dégradés du centre d'Athènes, à l'amélioration de leur image et à leur " occupation nouvelle " par les classes sociales «créatives» de la ville. Propositions qui font fi des processus sociopolitiques qui ont historiquement présidé à la formation de l'agglomération, négligent la réalité pénible et embrouillée actuelle et surtout foulent aux pieds toute procédure de participation ou d'implication des habitants dans la mesure où elles sont présentées comme la réponse allant de soi d'un récit qui

8 Parmi les projets récents, on compte la transformation en rue piétonne de l'avenue Panépistimiou, l'une des artères centrales les plus importantes de la ville, dont le sens est hautement historique et symbolique. La réalisation de ces travaux a fait l'objet d'un concours international d'architecture et, récemment, ont été publiées les études définitives proposant l'élaboration d'un parc linéaire au centre de la ville. Selon ses vecteurs d'exécution, ce "projet fera d'Athènes au milieu de la crise une métropole d'Europe contemporaine une capitale modèle qui émerge des profondeurs des siècles pour contempler l'avenir » (Re-think Athens 2014). Cette proposition a provoqué un débat très vif parmi les urbanistes et autres chercheurs de la ville. 
vise à leur remplacement par des habitants plus « appropriés ». C'est ainsi que les travaux de réhabilitation vont de pair avec la pratique gouvernementale des opérations d'expulsion par la police de tous ceux qui ne coöncident pas avec l'image embellie tant souhaitée'.

Directement tramées à l'injustice socio-spatiale, dans la mesure où elles façonnent des géographies précises allant du niveau local à l'espace mondial, les pratiques autoritaires de répression renforcent le retranchement tout en établissant la bipolarisation idéologique qui le sert. Il est possible à cet égard de dégager trois messages liés les uns aux autres, formellement exprimés tant dans les récits médiatiques que dans la politique appliquée. Le premier concerne l'assimilation de la sécurité avec la poursuite pénale des conséquences de l'infériorisation sociale, contrairement à la mission de prévention de l'Etat Social. Le deuxième message concerne l'assimilation du crime à certaines formes de criminalité qui ont lieu dans l'espace public urbain (telles que les effractions commises contre les véhicules, les vols, les destructions d'équipement urbain $)^{10}$ et par suite la culpabilisation des groupes sociaux et des espaces précis. Le troisième et plus important concerne la notion de démocratie. Les campagnes de sécurité semblent satisfaire le sentiment diffus d'insécurité (lequel est principalement causé par les réductions politiques de l'État social) grâce à leur mobilisation contre tout ce qui diverge ou diffère (voir Luigi Ferrajoli cité dans Yialketsis, 2010). Assimilant immigrés, sans-abris, drogués, prostituées, marchands ambulants ou même manifestants à des criminels, elles construisent un "nous " moral, exclusif, opposé aux « autres », représentant une menace. Cette bipolarisation oppose entre eux quartiers et voisinages. Elle oppose aussi les habitants « convenables », conviés à retourner au centre d'Athènes pour " gentrifier » les quartiers « dégradés », à celles et ceux qui aujourd'hui les habitent.

Dans une ville où le discours dominant cultive la peur de l'autre, accentuant discriminations et stigmatisations, les constructions médiatiques concernent ainsi un "nous » intact, xénophobe et sexiste qui exclut celles et ceux qui ne remplissent pas les conditions normatives du modèle familial

9 Également significative est la proposition récemment publiée par la municipalité d'Athènes, connue comme Plan d'intervention urbaine intégrale $(\Sigma \mathrm{OA})$, concernant le centre commercial et historique de la ville. Lors du rapport final, les dix-huit objectifs d'intervention étaient cristallisés en un programme d'action qui plaçait en priorité la lutte contre les infractions à la loi, les problèmes liés aux maisons de prostitution et les dangers que présente la concentration d'immigrés clandestins dans le centre de la ville.

10 Les statistiques concernant l'augmentation de la criminalité totalisent crimes mineurs de la rue et homicides. Ne sont pas comptés par exemple les scandales financiers, les actes arbitraires ou la violence policière, surtout contre les migrants et les activistes (Zarafonitou, 2009: 280). Il est en outre significatif que la foule des victimes des accidents de la route apparait rarement dans le bulletin de la "criminalité » (Morelle et Tardié, 2011) - il s'agit ici, statistiquement parlant, de la cause la plus importante des morts violentes et des blessures dans la ville $d$ 'Athènes. 
« grec » - une forme de catharsis sociale $q u$ 'adopte dans son discours public et ses pratiques Aube Dorée, «version de la virilité agressive qui revendique la place de chef en tant que porteuse du salut de la nation » (Avdela et Psarra, $2012: 5)^{11}$.

Dans les circonstances incertaines et imprévisibles de la crise, les liens socio-spatiaux se désagrègent au quotidien et certains souhaitent les voir remplacés par d'autres liens plus «appropriés », plus «purs » et traditionnellement admis. Dans l'Athènes des cohabitations multiples, la quête d'identités imaginaires conduit à la solution de facilité : la séparation entre un vague "nous » constamment confronté aux "autres », entité encore plus vague, laquelle acquiert pourtant des traits évidents, dès que ce " nous » dialogue avec l'idéologie néo-nazie.

\section{ATTACHEMENTS ORDINAIRES : LES ESPACES DE COEXISTENCE ET DE CONFLIT}

Parallèlement aux récits dominants qui «fabriquent» la ville en ghetto ou en paysage d'insécurité, les habitants de la ville tentent de se composer des quotidiennetés vivables au milieu des difficultés de la crise (et non seulement). Ils doivent survivre avec le rétrécissement des revenus et l'augmentation du coût de vie, le chômage souvent de longue durée, les réductions dans les services publics et/ou municipaux et dans un contexte urbain où les agressions racistes d'Aube Dorée font de la violence une affaire quotidienne ${ }^{12}$. Ces bouleversements du vivre en ville engendrent des tensions à plusieurs

11 Depuis 1945 et jusqu'à la fin des années 1980, dans tous les pays d'Europe, les partis d'extrême-droite sont de petits groupes marginaux composés de quelques irréductibles nostalgiques du fascisme. La situation change au cours de la décennie suivante, où commence l'ascension progressive qui amène nombre de partis d'extrême-droite, parmi lesquels Aube Dorée, à siéger dans les conseils européens. Sur l'organisation Aube Dorée et sur son action à Athènes, voir infra.

12 L'organisation Aube Dorée, fondée en 1985, mène depuis vingt ans une action continuelle et marquante en tant qu'association criminelle coupable d'agressions collectives, "à l'aide de couteaux, de gourdins et de barres de fer» contre les "ennemis de l'intérieur et de l'extérieur ", se soldant par des dizaines de victimes, principalement des étrangers mais aussi des jeunes gens "improprement vêtus », des étudiants, des syndicalistes, des activistes etc., rares étant les cas d'agressions qui parviennent devant les tribunaux. Depuis 2007, en tant que parti, Aube Dorée participe aux élections nationales. En 2010, aux élections municipales, elle a vu son pourcentage passer de $0,29 \%$ à $5,29 \%$, le résultat étant l'élection de son chef au conseil municipal. En 2012, elle fait son entrée à l'Assemblée avec 6,92\% et dix-huit sièges. L'objectif d'Aube Dorée est, une fois au pouvoir, d'appliquer les «lois de la nature » qui assurent la domination du plus fort, de "purger» la société de tous les membres "vicieux " qui la dirigent, mais aussi les «faibles » qui composent son peuple, car « le faible n'a pas de place dans la nature, car si les faibles dominaient il n'y aurait pas de vie ». Voir Frangoudaki, 2013 : 17-19, 53, 73 (les extraits cités entre guillemets sont empruntés au site de l'organisation, mentionnés dans idem). Voir en outre Psarras, 2012. 
niveaux, que l'on ressent de façons diverses et inégales dans les différents quartiers d'Athènes, dans la mesure où ils s'inscrivent dans des inégalités socio-spatiales préexistantes (de revenu, d'épargne, de niveau de prévoyance, etc.) (Vaiou, 2013).

Les habitants de la municipalité d'Athènes composent une mosaïque d'à peu près deux cents ethnies, dont $10 \%$ sont des migrants (ou d'origine migratoire), qui dans certains quartiers du centre d'Athènes dépassent les $20 \%{ }^{13}$. Après 1989 , les habitants ont pu constituer peu à peu dans ces quartiers des quotidiennetés entrelacées et des formes d'attachement qui entraînent la familiarisation avec la différence et l'altérité, comprennent des controverses et nécessitent un investissement énorme en temps et en travail effectué surtout par les femmes, tant migrantes que locales.

Ces formes d'attachement ne sont pas seulement locales, elles s'étendent aussi sur plusieurs échelles géographiques, le quartier apparaissant comme une échelle importante dans les récits des femmes et des hommes de nos enquêtes ${ }^{14}$. Ainsi que nous le verrons par la suite, le quartier devient une aire où les combats d'appartenance et d'appropriation se déroulent, mais il est aussi ce qui est en cause comme enjeu dans de tels combats. Nous puisons nos exemples dans deux quartiers du centre d'Athènes, Sepolia et Aghios Panteleimon, où les luttes pour la coexistence et la territorialité révèlent tant les processus conflictuels que la formation des attachements ordinaires.

Sepolia et Aghios Panteleimon sont des quartiers situés près du centre-ville, peu éloignés l'un de l'autre et possédant de très bonnes liaisons de transport en commun avec tout le tissu urbain : Sepolia à l'ouest et Aghios Panteleimon à l'est de la gare centrale, séparés par la voie de chemin de fer.

13 ELSTAT, recensement 2001, 2011. Durant la crise, des flux de réfugiés originaires des zones de guerre se sont ajoutés aux migrants déjà établis depuis les années 1990.

14 Voir Vaiou, Bacharopoulou, Tounta et al., 2007 ; Lafazani, Lykogianni et Vaiou, 2010. Voir aussi Vaiou, 2014. 


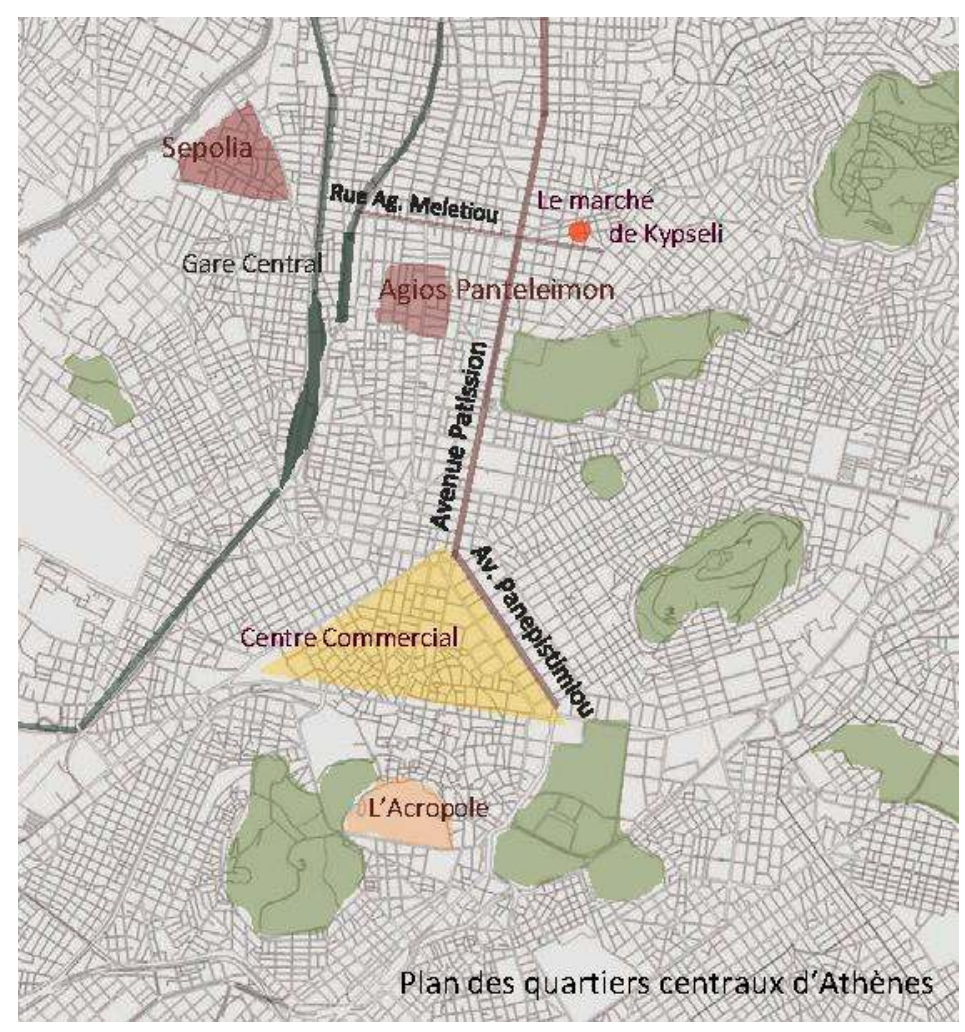

Plan des quartiers centraux d'Athènes avec les sites mentionnés dans le texte. Archive personnelle des auteurs

Sepolia était historiquement un quartier d'ouvriers, de migrants internes et de réfugiés d'Asie Mineure dans les années 1920, situé près de la zone industrielle d'Athènes (Elaionas), tandis qu'Aghios Panteleimon était habité par des représentants de la petite-bourgeoisie (salariés du secteur public, employés de banques, petits commerçants, étudiants et artistes...) (Leontidou, 1990). Après 1989, beaucoup de migrants ont trouvé des logements à loyer bon marché dans ces quartiers: les immigrés d'Albanie ${ }^{15}$ et $\mathrm{d}^{\prime}$ Europe de $\mathrm{l}^{\prime}$ Est dominent à Sepolia, mais on compte aussi la présence d'un petit groupe d'Africains (Égyptiens, Nigériens, Éthiopiens, Sudafricains) et d'un autre, encore plus restreint, d'immigrés d'autres pays du monde (les Philippines, l'Inde et le Pakistan). À Aghios Panteleimon, particulièrement depuis le début des années 2000, auprès des Albanais et des Européens de l'Est, on remarque la présence d'émigrés d'Afghanistan, du Pakistan et du Bangladesh, et même plus tard de Chine. Pendant plus de deux décennies, migrants et locaux ont trouvé des modes de vie commune et séparée à travers des pratiques quotidiennes et ordinaires et parfois à travers des activités organisées ensemble. Mais depuis 2008, les destins des deux quartiers divergent.

15 Les émigrés d'Albanie constituent le plus grand groupe d'immigrés en Grèce et à Athènes (plus de $55 \%$ de la population immigrée). Ils sont dispersés dans la ville, raison pour laquelle on les rencontre dans tous les quartiers. 
À Sepolia, l'embarras initial et l'hostilité silencieuse des locaux s'est lentement transformé en un type de coexistence où les contacts directs sont sélectifs et les cas d'agression ouverte rares et maîtrisés, comme le remarque Nikos, habitant de Sepolia depuis les années 1970 (interviewé en 2006), soulignant indirectement ses attachements d'autrefois :

les épiceries ont disparu du quartier. Maintenant on a des mini-market gérés par des étrangers. On a la boutique pakistanaise au coin, le magasin de vêtements chinois [...]. On rencontre des corps qui ne sont pas familiers, des gens pauvres et des gens plus à l'aise [...]. Maintenant on se souvient du quartier, on ne le voit plus.

Les divers habitants cherchent de nouveaux repères et développent des manières différentes de s'attacher dans le quartier qui change. Leurs pratiques quotidiennes et les rencontres informelles dans les espaces publics, dans les boutiques et les écoles du quartier mobilisent des mécanismes d'inclusion et des sentiments d'attachement. Nikos remarque :

dans les rues et sur les balcons on entend des langues qu'on ne comprend pas. Les femmes s'assoient sur les trottoirs, comme on faisait il y a longtemps, elles tricotent, elles parlent aux gens, les enfants jouent dans la rue. Mais elles parlent des langues que nous ne connaissons pas.

Les migrants recherchent des pratiques et des espaces urbains où se met en place une " citoyenneté des non-citoyens ", même fragile mais active dans des petits endroits de l'espace et des moments fugaces du temps (Painter et Philo, 1995 : 117 ; Kalandides et Vaiou, 2012). Anja, une migrante d'Ukraine, nous dit : « Vous savez, nous vivons tous ici, nous devons prendre soin de l'endroit. Moi, je suis allée à la mairie et je me suis plainte du parc. Il est en très mauvais état et nos enfants jouent là. » Elle affirme par-là ses propres attachements en faveur desquels elle s'active et revendique l'amélioration de l'espace public.

La crise évoluant, l'appauvrissement d'un grand nombre de personnes et de ménages est visible, à Sepolia comme ailleurs en ville; elle se lit à travers les vitrines toujours plus modestes, les boutiques fermées et sombres, le recours de beaucoup d'habitants à la soupe populaire proposée par l'Église ou des ONG, l'ouverture de bazars ou la réapparition de l'ardoise chez les épiciers locaux. Des effets moins visibles sont aussi repérables, tels que le démantèlement des services de proximité (pour les enfants ou les personnes âgées), ainsi qu'un nombre croissant d'élèves qui vont à l'école sans avoir pris de repas adéquat ${ }^{16}$. Ces difficultés sont souvent dépréciées puisqu'elles

16 Selon une récente enquête effectuée sur 6300 enfants des écoles publiques d'Athènes, publiée dans différents journaux, $23 \%$ des enfants vivent en-dessous du seuil de pauvreté et $14 \%$ dans des familles qui ne peuvent pas acheter la nourriture appropriée à leurs besoins ou payer leurs factures élémentaires. Voir par exemple les journaux Efimerida ton Syntakton 
affectent les «autres". Les conditions de coexistence sont confrontées à des tensions entretenues, sinon initiées, par des membres et partisans d'Aube Dorée qui se présentent comme les «vrais habitants" de Sepolia, même si beaucoup d'entre eux ne vivent même pas dans le quartier. Pourtant les expériences des deux décennies précédentes montrent que des attachements multiples limitent la portée des actes violents. Nous rencontrons de tels attachements au niveau des rencontres spontanées qui se déroulent sur les places et les jardins d'enfants, les escaliers de l'église où se concentrent des élèves après la classe, à l'entrée de l'école où les mamans se côtoient et discutent entre elles en attendant leurs enfants, dans les épiceries, minimarchés et boulangeries où se déroule la quotidienneté du quartier. Les rapports et les équilibres de coexistence se vérifient dans tous ces cas, mais restent encore assez fragiles.

À Aghios Panteleimon, on constate depuis 2008 une présence beaucoup plus visible et agressive d'Aube Dorée et, par conséquent, beaucoup plus de tensions dans la vie quotidienne des habitants et dans les espaces publics du quartier. Ce quartier est devenu une zone emblématique de revendication du contrôle du territoire par l'expulsion des migrants. En 2008, l' « installation » sur la place d'un nombre important de sans-papiers d'Afghanistan mit le feu aux poudres. Les tensions grandirent au point que la mairie d'Athènes ferma en mai 2009 l'aire de jeux qui fonctionnait comme un "centre d'accueil informel ", mais constituait aussi l'un des lieux du quotidien commun des habitants installés sur place ${ }^{17}$. C'est alors que les «comités de quartier » firent leur apparition, réclamant le départ des "immigrés clandestins », comités qui, ainsi qu'on le découvrit rapidement, avaient des relations directes avec Aube Dorée et ses « groupes de choc » (Kandylis et Kavoulakos, 2011).

Au cours de 2008 et 2009, les "comités de quartier » ou "les habitants enragés » ont organisé des manifestations et provoqué des agressions contre les migrants rendus coupables (des effets) de la crise ${ }^{18}:$ la chute des occasions d'emploi, celle des prix de l'immobilier, le déclassement des écoles locales, l'itinérance, l'insécurité, la maladie, les conditions épouvantables des espaces publics. Les pratiques violentes comprennent, entre autres, des coups et souvent des blessures graves portés aux migrants ainsi qu'aux citoyens opposants, des menaces envers les habitants qui emploient des migrants ou

20/11/2012, Avgi 25/12/2011, 12/12/2012, 25/12/2012, 31/1/2013, Kyriakatiki Eleftherotypia $16 / 10 / 2011$.

17 Les tensions existaient cependant depuis la période des Jeux Olympiques, lorsque de nombreuses piatsès, endroits où avaient lieu des pratiques illégales (prostitution, trafics divers dont celui de drogue, rackets et vols...), furent exclus des espaces où se déroulait cette manifestation internationale.

18 Quelques slogans d'Aube Dorée sont significatifs: "Tu es né grec, tu ne le deviens jamais »; " Ton sang, nous allons le verser, porc d'albanais »; "Dehors les étrangers. La Grèce appartient aux grecs »; " Hitler pour mille ans » ; "Cet école, ces quartiers appartiennent aux nationalistes » (slogan marqué au mur de l'école locale). 
leur louent des appartements, des attaques dirigées contre les magasins des migrants et leurs lieux de culte $^{19}$. Ces pratiques visent à imposer le contrôle du territoire et la création d'un quartier "sans migrants "; dans le même temps, elles visent à discipliner les habitants afin qu'ils tolèrent la violence et gardent le silence (voir aussi Wacquant, 2007; McLeod et Johnstone, 2011).

Les pratiques agressives sont aussi accompagnées de récits qui se font jour dans des « lettres ouvertes », des brochures et des dépliants locaux qui parlent d'invasion, de dégradation esthétique, morale, culturelle et sanitaire, d'insécurité, de perte des repères au niveau local où des lieux emblématiques sont maintenant occupés ou bien envahis par les «autres». Ces récits soulignent le passé du quartier comme étant " l'un des meilleurs quartiers d'Athènes ", aux cafés et restaurants réputés partout en ville, qui sont autant de repères désormais perdus pour les habitants ${ }^{20}$. De tels arguments sont aussi repris par les médias qui ont contribué à faire de Aghios Panteleimon un ghetto dangereux.

[L]es plus anciens habitants surtout se rappellent avec nostalgie les rues propres plantées d'arbres, leurs anciennes demeures bourgeoises à deux étages, on croirait qu'ils entendent encore les voix joyeuses des enfants remplir l'espace. Peu à peu le temps et la civilisation entre guillemets ont remplacé les vieilles et belles maisons par des immeubles monstrueux qui, cependant, continuent d'abriter ces familles bourgeoises bien connues, bien élevées et au mode de vie simple et propre ${ }^{21}$.

De tels récits dévoilent des attachements réels et fictionnels, d'un côté en omettant des « détails » significatifs, et de l'autre côté en confondant les temps et en transposant les lieux. Ici on peut citer beaucoup d'exemples : hormis la fuite volontaire des habitants vers les banlieues après 1980, le processus lucratif de la transformation des «demeures bourgeoises à deux étages » en immeubles; le vieillissement tant de la population que du parc immobilier (par exemple la transformation des "résidences à deux étages », qui n'étaient d'ailleurs pas si « bourgeoises » dans les années 1970, bien avant l'arrivée des immigrés); les familles bourgeoises habitant bien plus au nord de Aghios

19 Tous les gouvernements ont toléré ces pratiques, espérant pêcher des votes parmi les sympathisants et les militants de l'extrême-droite. Après l'assassinat du jeune musicien et activiste P. Fyssas par un membre d'Aube Dorée en septembre 2013, certains députés et cadres sont maintenant accusés d'actes criminels et certains d'entre eux, y compris le chef du parti, sont déjà en prison - ce qui a freiné l'activité extrême de cette organisation, du moins pour quelques temps.

20 Voir Kandylis, 2013 ; magazine « K» du journal Kathimérini du 13/1/2014 ("Quelque chose a-t-il changé à Aghios Pantéléimon? », pp. 40-46).

21 Z. B. "L'histoire de notre région », journal Foni ton katoikon, n¹, p. 8, février 2010, cité par Kandylis, 2013, p. 267. 
Panteleimon; leurs enfants ayant déserté depuis longtemps les écoles et les places du quartier.

Sur ce «champ de bataille», les menaces réelles ou imaginaires s'installent et influencent les relations socio-spatiales, complexes et conflictuelles. Les pratiques quotidiennes semblent maintenant façonnées par l'insécurité et la peur. Même les migrants bien installés dans le quartier depuis les années 1990 affrontent à leur tour avec des pratiques violentes les nouveaux arrivés pour défendre « leur » quartier et « leurs » boutiques. Les femmes de toutes ethnies hésitent à marcher dans les rues quand il fait noir. Les lieux de la violence deviennent un déni des différences qui se côtoient dans l'espace urbain, défiant ainsi la dimension fondamentale de «l'espace comme le site où se rencontrent, ou non, des trajectoires [différentes], où elles coexistent, s'influencent mutuellement, se battent » (Massey, 1999: 37).

\section{OBSERVATIONS FINALES}

La crise entraîne toutes sortes de bouleversements dans les manières d'être et de vivre la ville, dans la mesure où la précarité sociale et économique amène la montée du nationalisme, du racisme, du sexisme et de l'homophobie, de la violence contre les " autres » et généralement d'attitudes et de comportements plus conservateurs. Construire des attachements, vivre avec la multiplicité et l'engagement mutuel, avec une foule de trajectoires possibles et de choix de vie - constituant «un sens progressif du lieu», ainsi que Doreen Massey $(1994,2005)$ le soutient avec tant de force depuis de nombreuses années - n'est pas une conception exclusivement théorique. Il s'agit plutôt d'un processus de familiarisation avec la différence et l'altérité, qui comprend des controverses, nécessite un investissement en temps et en travail et peut conduire (ou pas) à des reformulations des attachements et de la façon dont ceux-ci se matérialisent (voir aussi Kalandides et Vaiou, 2012).

Les quartiers urbains présentent pour les habitants différentes chances de vie et opportunités d'inclusion (ou d'exclusion) dans la vie urbaine (Glick Schiller et Çaglar, 2009). Les quartiers de nos exemples sont des lieux mixtes où des personnes de diverses origines développent des routines quotidiennes se pratiquant au niveau local mais aussi liées à des processus supra-locaux, voire même mondiaux, qui se trament non seulement dans certains récits des migrants, mais aussi dans les activités locales, les boutiques et les produits, les odeurs et les sons, les usages de l'espace. Même si la coexistence ne conduit pas nécessairement à une mixité sociale, des relations de bon voisinage se développent néanmoins de manières diverses. Ces relations vont d'une familiarité avec les voisins à la formation de réseaux de solidarité et d'assistance mutuelle si importants dans la crise. 
En août 2012, le maire d'Athènes a mobilisé les Unités de Rétablissement de l'Ordre (MAT) afin de fermer le marché de Kypseli (situé tout près des deux quartiers choisis pour notre étude de cas). Selon son rapport au conseil municipal de la mairie d'Athènes $(27 / 8 / 2012)$ :

[...] Un des biens du citoyen athénien passe sous la responsabilité d'Athènes. C'était un de nos engagements devant le Conseil municipal et nous l'avons tenu : il n'est pas question de tolérer qu'un lieu public soit transformé en un lieu inaccessible. Nous donnerons la parole aux habitants de Kypseli pour le compte de ceux qui étaient les porte-paroles jusqu'à aujourd'hui. Après un dialogue où rien ne sera laissé de côté, nous allons décider ensemble et procéder à la réouverture du marché municipal, dans des termes d'entrée libre et de légalité [...].

Le marché municipal de Kypseli a été créé en 1935 et a fonctionné jusqu'à la fin du XXe siècle. En 2006, à l'initiative des habitants, il fut sauvé de la démolition et de sa transformation en parking à étages et fonctionna comme lieu public libre, ouvert et autogéré. Dans l'espace du marché ont été réalisées plus de 600 manifestations culturelles : représentations théâtrales, projections de films, concerts, expositions de peinture, cuisines collectives, fêtes des écoles publiques, bazars de charité, célébrations organisées par divers groupes d'habitants des différentes ethnies présentes dans la région, débats publics, soirées poétiques, présentations de livres, marchés de produits biologiques...

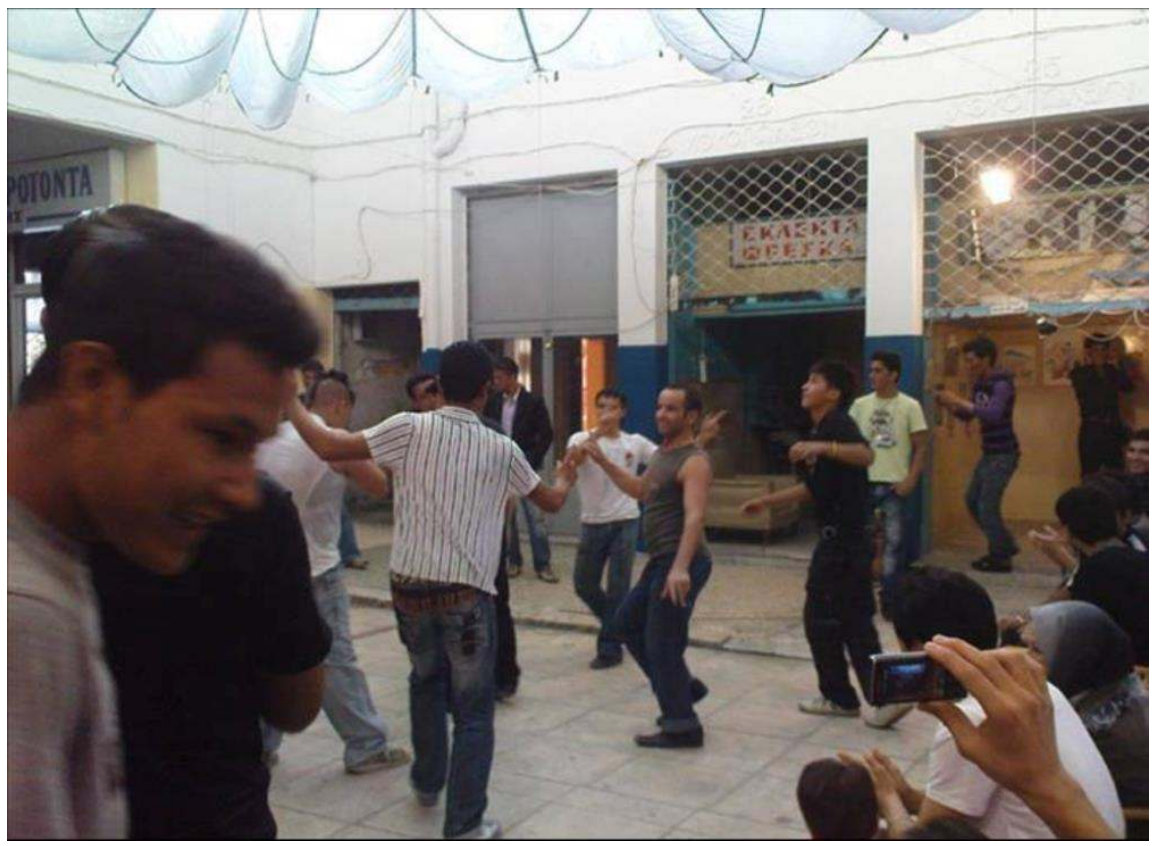

L` marché d’ Kyps`li, 2009. Fêt`organisé` par la communauté Afghan` d’ Grèc Archiv` p'rsonn`ll`d`s aut`urs 
Dans le cadre des activités du marché fonctionnait aussi quotidiennement une école d'enseignement de la langue grecque aux immigré(e)s, gérée par des instituteurs (trices) volontaires.

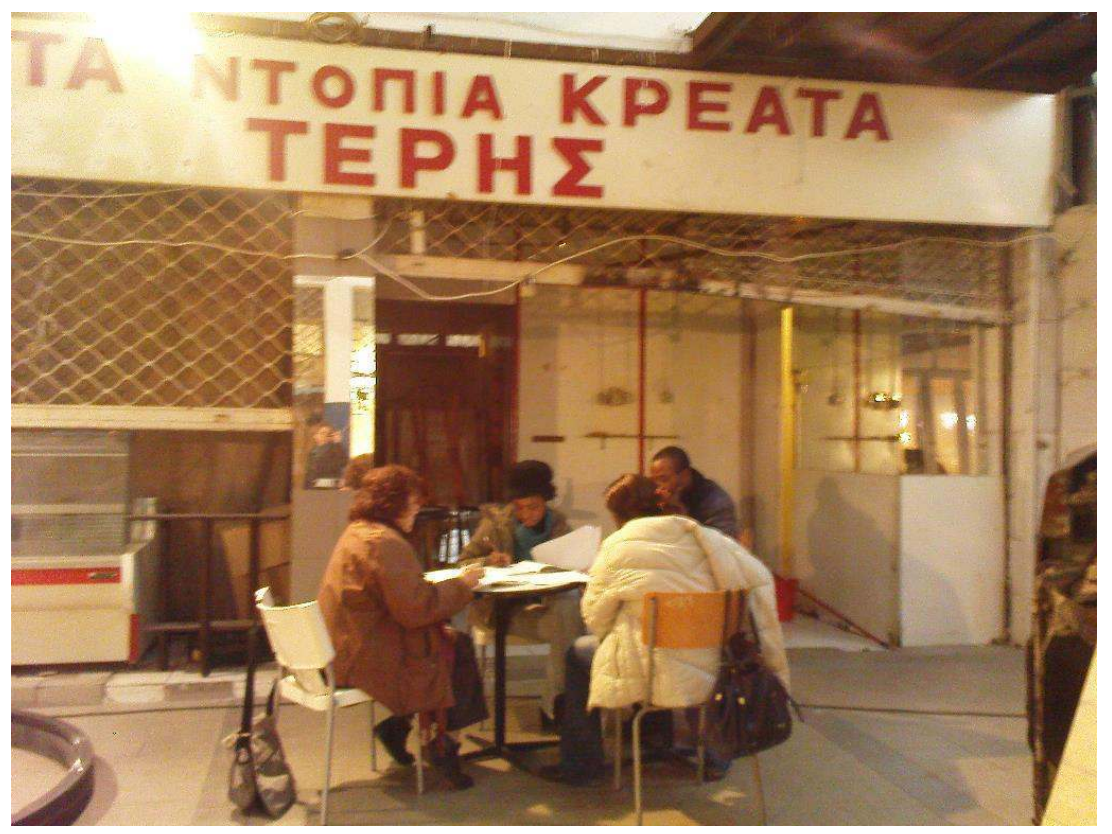

Le marché de Kypseli, 2009. L'école d'enseignement de la langue grecque aux immigré(e)s Archive personnelle des auteurs

Selon les propres termes d'une institutrice-informatrice volontaire, habitante de Kypseli, lors de l'une de nos enquêtes dans le quartier :

En dépit des problèmes qu'il rencontrait, le marché était quelque chose d'extrêmement important. C'est ainsi que des gens vivant dans le même quartier mais qui ne se connaissaient pas se sont rencontrés. Et qui avaient beaucoup de choses en commun [...] Le marché change pour moi le sens du quartier, je le sens plus familier, plus beau [...] L'école, eh bien, l'école, c'est plus pour apprendre nous-mêmes que pour que les immigrés apprennent. Apprendre qui nous sommes ainsi que la ville où nous vivons.

Au cours de ces années, le marché de Kypseli montre un lieu à travers lequel se produisent et s'éprouvent des attachements ordinaires dans la vie quotidienne des habitants du quartier. Il est aujourd'hui fermé, dans l'attente $\mathrm{du}$ « dialogue » entre le maire et des habitants « convenables».

Le discours et l'attitude adoptés par le gouvernement sur les quartiers d'Athènes, définissant les problèmes comme des problèmes locaux (par exemple le rassemblement excessif d'immigrés clandestins, les nombreuses autorisations d'ouverture de magasins accordées de façon incontrôlée à des étrangers à Aghios Panteleimon, le marché de Kypseli comme espace illégal), et faisant de leurs habitants des "autres-coupables», rend légitime la 
recherche de «solutions de protection et d'assainissement » locales. Un des traits caractéristiques de la violence d'Aube Dorée à Aghios Panteleimon (et ailleurs) est son caractère public : elle se déroule dans les rues, sur les places, dans des lieux de la ville exposés aux regards et auxquels chacun a accès (et non dans l'espace privé de l'habitation où la violence règne de toute façon) (Kandylis, 2013).

Depuis 2011, le «Réseau de l'Enregistrement de la Violence Raciste » retrace des événements de violence publique en Grèce (http://rvrn.org). A la micro-échelle de notre enquête, différents évènements de ce type surgissent, des attaques physiques telles que coups de poing, de pied et crachats contre un couple homosexuel, coups de couteau portés à un migrant par des membres d'Aube Dorée, menaces contre des organisateurs d'une pièce de théâtre pour enfants sur la place, passage à tabac d'une activiste, incendie volontaire d'un commerce tenu par un migrant pakistanais, harcèlement d'une professeure ayant parlé du nazisme à l'école. De tels évènements coexistent avec d'autres formes d'expression du rejet (par exemple le discours public sur le centre de la ville présenté en tant que « territoire perdu » comme lors d'opérations de guerre).

La coexistence comme une expérience du vivre avec la diversité permet aux voisins de bénéficier d'une certaine convivialité (Germain, 2002). Mais la coexistence ne garantit pas l'acceptation de la différence. Une communauté qui se sent menacée (par le chômage, la criminalité, la pauvreté) pourrait être plus encline à l'agression contre ce qui est proche et visible que contre ce qui paraît lointain (tels que, par exemple, les processus mondiaux qui amènent tant de migrants et de réfugiés en ville) ; elle serait probablement disposée à produire des récits d'exclusion des "autres», ainsi que l'indique notre exemple d'Aghios Panteleimon.

Les réalités quotidiennes vécues dans la ville sont plus compliquées que leurs images médiatiques. La présence policière qui va croissant depuis les années 1990 dans l'espace public d'Athènes et le discours de la classe politique ainsi que celui de la presse qui la sert, agissent sur la vie ordinaire dans les quartiers du centre d'Athènes en dressant la carte des "lieux inaccessibles ", des "ghettos d'étrangers » et des "zones à haute criminalité ». Là, des myriades d'attitudes et de pratiques, éloignées des feux de l'actualité, sont en quête de stratégies de survie et performent une culture différente de la vie quotidienne. À petits pas, difficiles mais opiniâtres, cette culture forme des relations plus substantielles et détermine à nouveau les attachements sociospatiaux. 


\section{BIBLIOGRAPHIE}

Vassilis ARAPOGLOU et Thomas MALOUTAS, «Segregation, inequality and marginality in context: The case of Athens », The Greek Review of Social Research, 136, 2011, pp. 135-155.

Efi AVDELA et Aggelika PSARA, " Aspects cachés de l'encre du vote », Synchrona Themata, 117, avril - juin, 2012, pp. 4-5 (en grec).

Zygmunt BAUMAN, Liquid Times. Living in an Age of Uncertainty, Cambridge, Policy Press Ltd, 2007 (traduction grecque 2008).

Bianca BOTEA, "Expérience du changement et attachements. Réaménagement urbain dans un quartier lyonnais (la Duchère) », Ethnologie française, 44, pp. 461-467, 2014.

Anna FRANGOUDAKI, Le nationalisme et la montée de l'extrême-droite, Athènes, Alexandréia, 2013 (en grec).

Annick GERMAIN, «The social sustainability of multicultural cities: a neighbourhood affair? », BELGEO, 4, 2002, pp. 377-386.

Nina GLICK SCHILLER et Ayşe CAGLAR, "Towards a comparative theory of locality in migration studies. Migrant incorporation and city scale », Journal of Ethnic and Migration Studies, 35 (2), 2009, pp. 177-202.

Stuart HALL, Doreen MASSEY et Michael RUSTIN, « After neoliberalism: analyzing the present», [en 2013, ligne]. http:/www.lwbooks.co.uk/journals/soundings/manifesto.html

Antoine HENNION, " D'une sociologie de la médiation à une pragmatique des attachements », Sociologies, Théories et recherches, 2013 [en ligne]. URL : http://sociologies.revues.org/4353

Ares KALANDIDES et Dina VAIOU, " "Ethnic" neighbourhoods? Practices of belonging and claims to the city », European Urban and Regional Studies, 19 (3), 2012, pp. 254-266.

George KANDYLIS, "L'espace et le temps du rejet des immigrés dans le centre d'Athènes ", in Thomas MALOUTAS et al. (dir.), Le centre d'Athènes en tant qu'enjeu politique, Athènes, EKKE, 2013, pp. 257-279 (en grec).

George KANDYLIS et Karolos I. KAVOULAKOS, « Framing urban inequalities: racist mobilization against immigrants in Athens », Greek Review of Social Research, 136C, 2011, pp. 157-176.

George KANDYLIS, Thomas MALOUTAS et John SAYAS, «Immigration, inequality and diversity: socio-ethnic hierarchy and spatial organization in Athens, Greece ", European Urban and Regional Studies, 19/3, 2012, pp. 267-286.

Olga LAFAZANI, Rouli LYKOGIANNI et Dina VAIOU, Urban Interaction Spaces and Social Movements, National Report from Greece in the context of GeMIC (Gender, migration and intercultural interaction in the Mediterranean and South-East Europe. An interdisciplinary perspective), FP7 projet de recherche sous la coordination de Maria STRATIGAKI, Université Panteion, Grèce, 2010 (www.gemic.eu). 
Lila LEONTIDOU, The Mediterranean City in Transition, Cambridge, Cambridge University Press, 1989.

Doreen MASSEY, Space, Place and Gender, Cambridge, Polity Press, 1994.

Doreen MASSEY, Power Geometries and the Politics of Space-Time, Heidelberg, Department of Geography, University of Heidelberg, Hettner Lecture, 1999.

Doreen MASSEY, For Space, Londres, Sage, 2005.

Irène MICHA, "L'impact de la politique de sécurité en ville sur les multiples significations de l'espace public », Synchrona Themata, 98, juillet - septembre, 2007, pp. 81-87 (en grec).

Gordon MCLEOD et Craig JOHNSTONE, « Stretching urban renaissance: privatising space, civilising place, summoning community », International Journal of Urban and Regional Research, 36(1), 2012, pp. 1-28.

Marie MORELLE et Jérôme TARDIE, « Pratiques de sécurité en ville », Justice Spatiale, 4, 2012 [en ligne]. URL : http://jssj.org/index.php

Chantal MOUFFE, On the Political, Abingdon - New York, Routledge, 2005, (traduction grecque 2010).

Jo PAINTER et Chris PHILO, «Spaces of citizenship: an introduction », Political Geography, 14 (2), 1995, pp.107-120.

Dimitris PSARRAS, Le Livre noir d'Aube Dorée, Athènes, Polis, 2012 (en grec).

Dina VAIOU, «Transnational city lives: changing patterns of care and neighbouring in Athens ", in Linda PEAKE et Martina RIEKER (dir.), Rethinking Feminist Interventions in Urban Studies, New York, Routledge, 2013, pp. 52-67.

Dina VAIOU, «Les aspects genrés de la crise à Athènes », Cahiers du CEDREF, 19, 2012, pp. 99-120.

Dina VAIOU, "Is the crisis in Athens (also) gendered? Facets of access and (in)visibility in everyday public spaces », City, 18(4-5), 2014, pp. 533-537.

Dina VAIOU, Anna BACHAROPOULOU, Fotini TOUNTA (et al), Quotidiennetés entrelacées et transformations socio-spatiales dans la ville. Des femmes migrantes et locales dans les quartiers d'Athènes, Athènes, L-Press et NTUA, 2007 (en grec).

Loïc WACQUANT, "Territorial stigmatization in the age of advanced marginality », Thesis Eleven, 91, 2007, pp. 66-77.

Thanassis YIAKELTSIS, «La peur en tant qu'instrument politique », Kyriaktaiki Elefthérotypia et 7 I techni tis zois, 14 novembre, 2010, pp. 22 (en grec).

Christina ZARAFONITOU, «Criminal Victimisation in Greece and the Fear of Crime: A "Paradox" for Interpretation », International Review of Victimology, 16/3, 2009, pp. 277-300.

Christina ZARAFONITOU, « De la justice de réparation à la justice de réhabilitation : esprit de coercition ou émoussement des conflits?», in A. GIOTOPOULOUMARAGKOPOULOU (dir.), La criminologie face aux défis contemporains. Congrès anniversaire des 30 années de la Société hellénique de Criminologie, édition Nomiki Vivliothiki, Athènes, 2011, pp. 115-129 (en grec). 
RÉSUMÉ : La crise de la société grecque a provoqué des changements radicaux qui s'accompagnent de violents bouleversements sociaux et de transformations urbaines brutales. L'intention de cet article est d'explorer les changements en cours, en se focalisant sur les conflits et les mutations des attachements, d'une part quotidiens, c'est-à-dire produits par de multiples pratiques socio-spatiales fréquemment conflictuelles mais aussi dynamiques, et d'autre part construits par un discours moral (dit politique) favorisé par les médias qui, ces dernières décennies, domine sur la vie $\mathrm{d}^{\prime}$ Athènes. Notre analyse s'appuie sur une recherche qui vise la constitution de ce discours, ainsi que les pratiques quotidiennes moins visibles et les modes d'appropriation de l'espace urbain par tous ceux que le discours dominant exclut.

MOTS-CLÉS : attachements, discours médiatique, pratiques quotidiennes, sécurité, quartiers du centre d'Athènes. 